Article

\title{
Individualized Meal Replacement Therapy Improves Clinically Relevant Long-Term Glycemic Control in Poorly Controlled Type 2 Diabetes Patients
}

\author{
Kerstin Kempf ${ }^{1,+}$, Martin Röhling $\left.{ }^{1, *,+}{ }^{(}\right)$, Katja Niedermeier ${ }^{1}$, Babette Gärtner ${ }^{1}$ and \\ Stephan Martin 1,2 \\ 1 West-German Centre of Diabetes and Health, Düsseldorf Catholic Hospital Group, Hohensandweg 37, 40591 \\ Düsseldorf, Germany; kerstin.kempf@wdgz.de (K.K.); katjaniedermeier@web.de (K.N.); \\ babette.gaertner@vkkd-kliniken.de (B.G.); stephan.martin@vkkd-klinken.de (S.M.) \\ 2 Faculty of Medicine, Heinrich Heine University Düsseldorf, 40225 Düsseldorf, Germany \\ * Correspondence: martin.roehling@vkkd-kliniken.de; Tel.: +49-(0)211-56-60-360-76; \\ Fax: +49-(0)211-56-60-360-72 \\ + Equal authorship.
}

Received: 14 June 2018; Accepted: 1 August 2018; Published: 4 August 2018

Abstract: Background Formula diets can improve glycemic control or can even induce remission in type 2 diabetes. We hypothesized that especially an individualized intense meal replacement by a low-carbohydrate formula diet with accompanied self-monitoring of blood glucose (SMBG) contributes to long-term improvements in $\mathrm{HbA1c}$, weight, and cardiometabolic risk factors in poorly controlled type 2 diabetes. Methods Type 2 diabetes patients were randomized into either a moderate group (M-group) with two meal replacements/day $(n=160)$ or a stringent group (S-group) with three meal replacements/day $(n=149)$ during the first week of intervention (1300-1500 kcal/day). Subsequently, both groups reintroduced a low-carbohydrate lunch based on individual adaption due to SMBG in weeks 2-4. After week 4, breakfast was reintroduced until week 12. During the follow-up period, all of the participants were asked to continue replacing one meal per day until the 52-weeks follow-up. Additionally, an observational control group $(n=100)$ remained in routine care. Parameters were compared at baseline, after 12 and 52 weeks within and between all of the groups. Results 321 participants (83\%) completed the acute meal replacement phase after 12 weeks and 279 participants $(72 \%)$ the whole intervention after 52 weeks. Both intervention groups achieved improvements in $\mathrm{HbA1c}$, fasting blood glucose, blood pressure, and weight (all $p<0.001$ ) within 12 weeks. However, these results were not significantly different between both of the intervention groups. The estimated treatment difference in $\mathrm{HbA} 1 \mathrm{c}$ reduction was (mean $(95 \%$ confidence interval $[C I])-0.10 \%$ with $95 \% \mathrm{CI}[-0.40 ; 0.21]$ also $(p>0.05)$ (S-group vs. M-group) not statistically different after 12 weeks. However, only the S-group showed a clinically relevant improvement in $\mathrm{HbA1c}$ of $-0.81 \%[-1.06 ;-0.55](p<0.001)$ after 52 weeks of follow-up, whereas HbA1c was not statistically different between the $\mathrm{M}$ - and control group. Conclusion Individualized meal replacement with SMBG demonstrated beneficial effects on $\mathrm{HbA} 1 \mathrm{c}$ and cardiometabolic parameters in type 2 diabetes. Furthermore, the initiation of a weight loss program with one week of full meal replacement (three meals per day) resulted in a clinically relevant long-term $\mathrm{HbA1c}$ reduction, as compared to an observational control group that had standard care.

Keywords: type 2 diabetes; low-carbohydrate diet; $\mathrm{HbA1c}$; weight loss; formula diet 


\section{Introduction}

Current type 2 diabetes mellitus guidelines recommend lifestyle intervention as basic treatment. However, patients often fail to improve their eating behavior, physical activity, body weight, and glycemic control in the long run. In this context, new strategies have been developed, such as technology-based approaches [1], to improve adherence to lifestyle interventions and to enable long-term benefits [2]. In contrast, a failing lifestyle intervention contributes to an initiation of a pharmaceutical co-intervention in the next step, however, anti-diabetic medication does not prevent the progression of type 2 diabetes [3]. Within 10 years after diagnosis, about $50 \%$ of type 2 diabetes patients start with insulin therapy [4]. This often results in additional weight gain, leading to an increased insulin dosage [5]. Thus, this vicious circle proceeds and disease remission had been unlikely until bariatric surgery demonstrated that type 2 diabetes is reversible [6]. After bariatric surgery, glycemic control improves within a few days, even before a decrease of body weight becomes apparent [7], but this treatment has several severe side-effects [8] and long-term effects are still unclear [9]. In this context, it is still unknown whether the magnitude of improvement is primarily due to caloric restriction or is unique to the surgical procedure [10]. Given the huge need for alternative approaches with long-term effects regarding $\mathrm{HbA} 1 \mathrm{c}$ reduction and remission of diabetes, formula diets can be simple and effective measures [11]. Furthermore, the use of energy-restricted formula diets in obese persons with type 2 diabetes improved cardiometabolic endpoints, e.g., waist circumference, fat mass, blood pressure, insulin, or $\mathrm{HbA1c}$, [12]. Moreover, intervention studies, especially those from a group in the United Kingdom (UK) [11,13-15], with a stringent and very low-calorie formula diet were even able to induce diabetes remission [13-15]. In previously published studies, we had already investigated the single or combined effect of low-carbohydrate formula diets and/or telemedicine in patients with type 2 diabetes inducing $\mathrm{HbA1c}$, anti-diabetic medication, and body weight improvements [12,16]. Furthermore, we could also demonstrate the beneficial effect of individual meal prescription accompanied with self-monitoring of blood glucose (SMBG) in patients with type 2 diabetes [17]. However, there are hardly any studies investigating the dose-response relationship of an early intense and individualized low-carbohydrate and moderate-calorie meal replacement therapy by formula diet in patients with type 2 diabetes. Furthermore, a previous study revealed a high dropout rate of $32 \%$ for a stringent diet intervention with low-carbohydrate meal replacement [12]. We, therefore, conducted the current intervention by comparing two diet regimens, differing in treatment intensity, with a third observation control group that remained in routine care, in patients with type 2 diabetes.

\section{Materials and Methods}

\subsection{Study Design}

The present study consisted of two intervention groups and one observational control group. Volunteers were recruited in Germany by newspaper articles. Eligible type 2 diabetes patients were randomized according to an electronically generated randomization list into two parallel intervention groups with either a moderate (M-group, $n=160$ ) or a stringent diet regime (S-group, $n=149$ ). The observational group $(n=100)$ corresponds with the control group from our TeLiPro study (NCT02066831) [16]. The participants, the study nurse, and the outcome assessor were blinded for sequence of allocation concealment. The first participant was enrolled on 7 February 2012 and the last participant finished the intervention on 13 June 2014. The study was conducted at the West-German Centre of Diabetes and Health in Düsseldorf (WDGZ), Germany, in cooperation with family doctors and diabetologists around Germany and in accordance with the ethical standards that were laid down in the 1964 Declaration of Helsinki and its later amendments. Approval of the research protocol was obtained from the ethics committee of the Ärztekammer Nordrhein (No. 2011294) and it was registered at clinicaltrials.gov under the number NCT02230501, ClinicalTrials.gov. All of the participants gave written informed consent prior to their inclusion into the study. 


\subsection{Study Population}

Patients with type 2 diabetes, aged 25-79 years with poorly controlled glucose levels $(\mathrm{HbA} 1 \mathrm{c} \geq 7.5 \%)$, and body mass index $(\mathrm{BMI}) \geq 27 \mathrm{~kg} / \mathrm{m}^{2}$ were included in the study. Participants were excluded when one of the following exclusion criteria was existent: (i) acute infections; (ii) chronic diseases such as cancer, chronic obstructive pulmonary disease, asthma, dementia, chronic gut diseases, psychoses, liver cirrhosis, nephropathy, and kidney insufficiency with glomerular filtration rate $<30 \mathrm{~mL} / \mathrm{min} / 1.73 \mathrm{~m}^{2}$; (iii) weight loss of $>2 \mathrm{~kg} /$ week in the last month; (iv) smoking cessation or planned smoking cessation during the study; (v) drugs for active weight reduction; (vi) pregnancy or breast-feeding; and, (vii) known intolerance with components of the used formula diet.

\subsection{Intervention}

At the first contact, the design and intention of the study were explained to the participants by study nurses and trial physicians. A manual and a formula diet were handed out to the patients of the intervention groups. The manual included information about the preparation of the individualized meal replacement as well as general facts about low-carbohydrate meals and their interaction with the blood glucose level. Participants were instructed to perform self-monitoring of blood glucose (SMBG) and note down these values into the manual, the amount of meal replacement taken, the number of meals replaced, as well as their daily dose of anti-diabetic medication. Participants were advised to perform a seven-point blood glucose diurnal profile and they were urged to perform event-driven measurements, e.g., 1.5-2 h after no, low-, or high- carbohydrate consumption or in the fasting state in the morning when exercise had been done the evening before. The patients were encouraged to draw their own conclusions from the SMBG results and to adapt their meals and habits aiming to keep blood glucose levels within a normal range, which was individually prescribed and adapted during the study process. The manual provided guidance on how to change eating habits and how to react to elevated blood glucose levels with physical activity. Based on their own experience and in accordance with the prescriptions to adapt their blood glucose levels, participants were responsible for modifying their diet and received help in the case of nutrition-related uncertainties. In sum, meal replacement and SMBG were individually recommended and adopted to the personal preferences throughout the study. Based on these values, anti-diabetic therapy was monitored and then individually adjusted by trial physicians. This "personalized nutrition and treatment" was one of the main educative approaches in our study. At each visit, study nurses revised the manual and educated/instructed the participants in terms of low-carbohydrate diet, SMBG, physical activity, and self-motivation. Study visits took place after week 1, 4, and 12 and were accompanied with telephone calls or personal meetings. A detailed timeline of the study visits is shown in the Supplementary Figure S1. Participants of the control group only received a self-management guide, a weighing scale, as well as a step counter and they were advised to measure their steps and weight daily.

\subsection{Outcomes and Measurements}

Clinical and biochemical data were measured at baseline, after 12 weeks of intervention, and after 52 weeks of follow-up. Venous blood was collected after an overnight fast and abdication of medication of at least $10 \mathrm{~h}$ by inserting an intravenous cannula into the forearm vein, and laboratory parameters ( $\mathrm{HbA} 1 \mathrm{c}$, fasting blood glucose, total cholesterol, high-density-lipoprotein (HDL), and low-density-lipoprotein (LDL) cholesterol) were analyzed at the local laboratory as described in detail elsewhere [16]. Validated questionnaires were used to assess eating behavior (German version of the 'Three-factor Eating Questionnaire' (TFEQ)) and quality of life ('Short Form-36' (SF36)), as previously described [16]. Anti-diabetic medication and changes throughout the study were documented. Adverse events were documented. 


\subsection{Diet Regimen}

The chosen formula diet (Almased-Vitalkost; Almased-Wellness-GmbH, Bienenbüttel, Germany) contained $30.6 \mathrm{~g}$ carbohydrates and $1507 \mathrm{~kJ}$ ( $360 \mathrm{kcal})$ energy per $100 \mathrm{~g}$ powder and it was provided to all study probands during the whole study period. Participants of the intervention groups replaced breakfast, lunch, and dinner with $1 \mathrm{~g}$ Almased $/ \mathrm{kg}$ normal body weight (defined as height in $\mathrm{cm}$ -100) per meal dissolved in $250 \mathrm{~mL}$ water during the first week and consumed $45 \mathrm{~g}$ of oil rich in omega-3-fatty acids ( $1665 \mathrm{~kJ} ; 398 \mathrm{kcal})$ and $750 \mathrm{~mL}$ vegetable juice each day, as previously described [16]. No additional snacks were permitted. During weeks $2-4$, the participants replaced breakfast and dinner with the formula diet and ate a low-carbohydrate lunch. The lunch should include 150-200 g of fish or meat, $500 \mathrm{~g}$ vegetables, and not more than $50 \mathrm{~g}$ of carbohydrates from wholegrain bread or brown rice. The low-carbohydrate nutrition had to be continued in the weeks 5-12, while only dinner was replaced by formula diet. Instructions were identical for the participants of both groups. The only difference between both intervention groups was that the M-group should only replace two meals per day during the first week. All of the participants were asked to continue replacing one meal per day during the follow-up period until the final visit at the 52-weeks follow-up. Both participant and study staff were responsible for the individualized treatment. SMBG as well as the personalized formula diet and the reintroduction of normal meals were interactively modified. Furthermore, the personalized formula diet depends on the current weight of each proband and is characterized by low-carbohydrate meals that are aiming to regulate a normal blood glucose level. We assessed protocol compliance by requiring the participants to note the frequency and amount of formula diet they used as well as the composition of their meals during the first 12 weeks. This information had to be sent back. Afterwards, they got another ration of formula diet for the next weeks. We chose this design with a very similar intervention program as the current study situation reveals that only intense behavioral lifestyle interventions can contribute to meaningful results [14], and we were interested in the dose-response pattern in initial treatment phase during the first week. Furthermore, we had seen in a previous study that a very stringent regime leads to high dropout rates, and we, therefore, wanted to test a gentler entry [12]. The control group remained in routine care (quarterly visits with their attending physician for routine health-care visits, as defined by the Disease Management Programs (DMP) for Type 2 Diabetes in Germany) and did not participate in the meal replacement program.

\subsection{Statistics}

Previous own data have indicated that with the use of a low-carbohydrate meal replacement a reduction in $\mathrm{HbA} 1 \mathrm{c}$ of $0.7 \%$ could be achieved [12], while a reduction of $1.0 \pm 0.8 \%$ for the S-group was assumed. To be able to measure differences between both of the intervention groups with a power of $80 \%$ and a level of significance of $5 \%$, a sample size calculation revealed that at least 230 datasets would be needed. Since a dropout rate of about $25 \%$ was estimated, the plan was to recruit a total of 140 participants per group. Data are presented as means and standard deviations (mean \pm SD), median and first and third quartiles (median (first; third quartiles)), means and 95\% confidence intervals (mean $[95 \% \mathrm{CI}]$ ), or percentages, as appropriate. Completer analyses were performed. Missing values were imputed by the 'last-observation-carried-forward' (LOCF) principle. As HbA1c is the primary parameter in the present study, LOCF was solely applied for other parameters.

Primary endpoint was the differences in Hba1c after 12 weeks between groups, secondary outcomes were the differences in body weight, BMI, cardiometabolic risk factors, eating behavior, quality of life, and frequency of anti-diabetic medication after 12 weeks of meal replacement intervention and 52 weeks of follow-up between the two intervention groups. Furthermore, the estimated treatment difference (ETD), as well as the proportion of weight loss in percentage, was determined. Non-parametric data were analysed with Mann-Whitney U, Wilcoxon, and Friedman test and parametric data with Student's $t$-test, paired $t$-test, and analysis of variance with repeated measures to determine the differences between groups following the intervention. Multivariable univariate regression analyses were carried out to investigate group differences while adjusting 
for baseline parameters. Dichotomous variables as well frequencies were compared by the Fishers exact test, McNemar test, or Cochrane $\mathrm{Q}$ test.

Tertiary outcomes focused on changes in all aforementioned parameters from baseline to week 12 and week 52 within both intervention groups. These were analyzed while using mixed models adjusting for repeated measurements, baseline values, and multiple testing.

Further analyses focused on differences between the intervention groups and the observational control group in regard to $\mathrm{HbA} 1 \mathrm{c}$ and weight loss. These analyses were performed in accordance with the statistical approaches used for the determination of the primary endpoints. All statistical tests were two sided, and the level of significance was set at $\alpha=0.05$. $P$ values were adjusted for multiple comparisons using Bonferroni correction. All of the analyses were performed using SPSS 22.0 (SPSS Inc., Chicago, IL, USA) and GraphPad Prism 6.04 (GraphPad Software, San Diego, CA, USA).

\section{Results}

A total of 309 participants were randomized into the S-group $(n=149)$ or M-group $(n=160)$, and a control group of $n=100$ were observed, as shown in Figure 1 . Three hundred and twenty-one participants (83\% [321:385], $n=125 \mathrm{M}$-group; $n=122 \mathrm{~S}$-group; $n=74$ control group) from the starting cohort finished the 12-weeks intervention, while 64 participants dropped out within the 12-weeks period. Follow-up data after 52 weeks were available from 279 participants (72\% [279:385]). Reasons for dropouts were: (i) spontaneous intolerances (5\%); (ii) health problems (25\%); (iii) professional reasons (5\%); (iv) personal reasons (60\%); and, other reasons (5\%). The demographical and clinical characteristics of the three groups are shown in Table 1. Participants who completed the intervention and follow-up phase and those who dropped out or were lost to follow-up did not differ significantly, apart from differences in diabetes duration, eating behavior, and quality of life between the groups (Supplementary Table S1). No adverse effects have been reported. Patients of the control group were more frequently treated with antidiabetic medication than those in the intervention groups, particularly, regarding insulin therapy. The individual antidiabetic drug classes are listed in Supplementary Table S2.

Table 1. Baseline characteristics of the participants who finished the 12-week diet intervention.

\begin{tabular}{|c|c|c|c|}
\hline & M-Group ( $n=125$ ) & S-Group ( $n=122)$ & Control Group $(n=74)$ \\
\hline Sex (\% male) & 46.4 & 52.5 & 52.7 \\
\hline Age (years) & $60 \pm 10$ & $59 \pm 9$ & $60 \pm 8$ \\
\hline Weight (kg) & $110 \pm 24$ & $107 \pm 20$ & $111 \pm 21$ \\
\hline BMI $\left(\mathrm{kg} / \mathrm{m}^{2}\right)$ & $37.5 \pm 7.6$ & $36.1 \pm 5.9$ & $37.0 \pm 6.7$ \\
\hline $\mathrm{HbA} 1 \mathrm{c}(\%)$ & $8.4 \pm 1.1$ & $8.4 \pm 1.2$ & $8.2 \pm 1.2$ \\
\hline Known diabetes duration (years) & $9 \pm 6$ & $8 \pm 7$ & $11 \pm 8^{\ddagger, \ddagger}$ \\
\hline $\mathrm{FBG}(\mathrm{mg} / \mathrm{dL})$ & $181 \pm 53$ & $178 \pm 63$ & $179 \pm 54$ \\
\hline $\mathrm{SBP}(\mathrm{mmHg})$ & $135 \pm 17$ & $134 \pm 14$ & $134 \pm 13$ \\
\hline $\mathrm{DBP}(\mathrm{mmHg})$ & $82 \pm 8$ & $80 \pm 8$ & $81 \pm 9$ \\
\hline Total cholesterol (mg/dL) & $200 \pm 52$ & $198 \pm 43$ & $194 \pm 48$ \\
\hline $\mathrm{HDL}(\mathrm{mg} / \mathrm{dL})$ & $46 \pm 10$ & $47 \pm 11$ & $47 \pm 11$ \\
\hline $\mathrm{LDL}(\mathrm{mg} / \mathrm{dL})$ & $118 \pm 32$ & $119 \pm 37$ & $117 \pm 36$ \\
\hline Triglyceride (mg/dL) & $383 \pm 586$ & $220 \pm 157$ & $194 \pm 113$ \\
\hline TFEQ [cognitive control] (au) & $10(7 ; 13)$ & $10(7 ; 13)$ & $7(6 ; 8){ }^{\mathrm{H}}$ ，㧊 \\
\hline TFEQ [suggestibility] (au) & $7(5 ; 10)$ & $7(4 ; 10)$ & 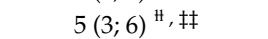 \\
\hline TFEQ [hunger] (au) & $6(4 ; 9)$ & $5(3 ; 9)$ & $5(4 ; 8)$ \\
\hline SF36 [physical health] (au) & $42(35 ; 50)$ & $42(34 ; 51)$ & $40(31 ; 52)$ \\
\hline SF36 [mental health] (au) & $49(38 ; 57)$ & $49(32 ; 57)$ & $39(35 ; 42){ }^{\mathrm{Ht}}$ ，执 \\
\hline
\end{tabular}

Shown are means $\pm \mathrm{SD}$, median (1st; 3rd quartiles) or percentages. ${ }^{\sharp} \mathrm{CON}$ vs. M-group, $p<0.01 ;{ }^{\ddagger} \mathrm{CON}$ vs. M-group, $p<0.05$; 㧊 CON vs. S-group, $p<0.01 ; \ddagger$ CON vs. S-group, $p<0.05 ;$ au, arbitrary units; FBG, fasting blood glucose; BMI, body mass index; DBP, diastolic blood pressure; HDL, high-density-lipoprotein; LDL, low-density-lipoprotein; SF36, short form-36; SBP, systolic blood pressure; TFEQ, three-factor eating questionnaire. 
Modified CONSORT flow diagram for individual randomized controlled trials of nonpharmacologic treatments.

An extra box per intervention group relating to care providers and centers has been added.

$\mathrm{IQR}=$ interquartile range; $\max =$ maximum; $\min =$ minimum

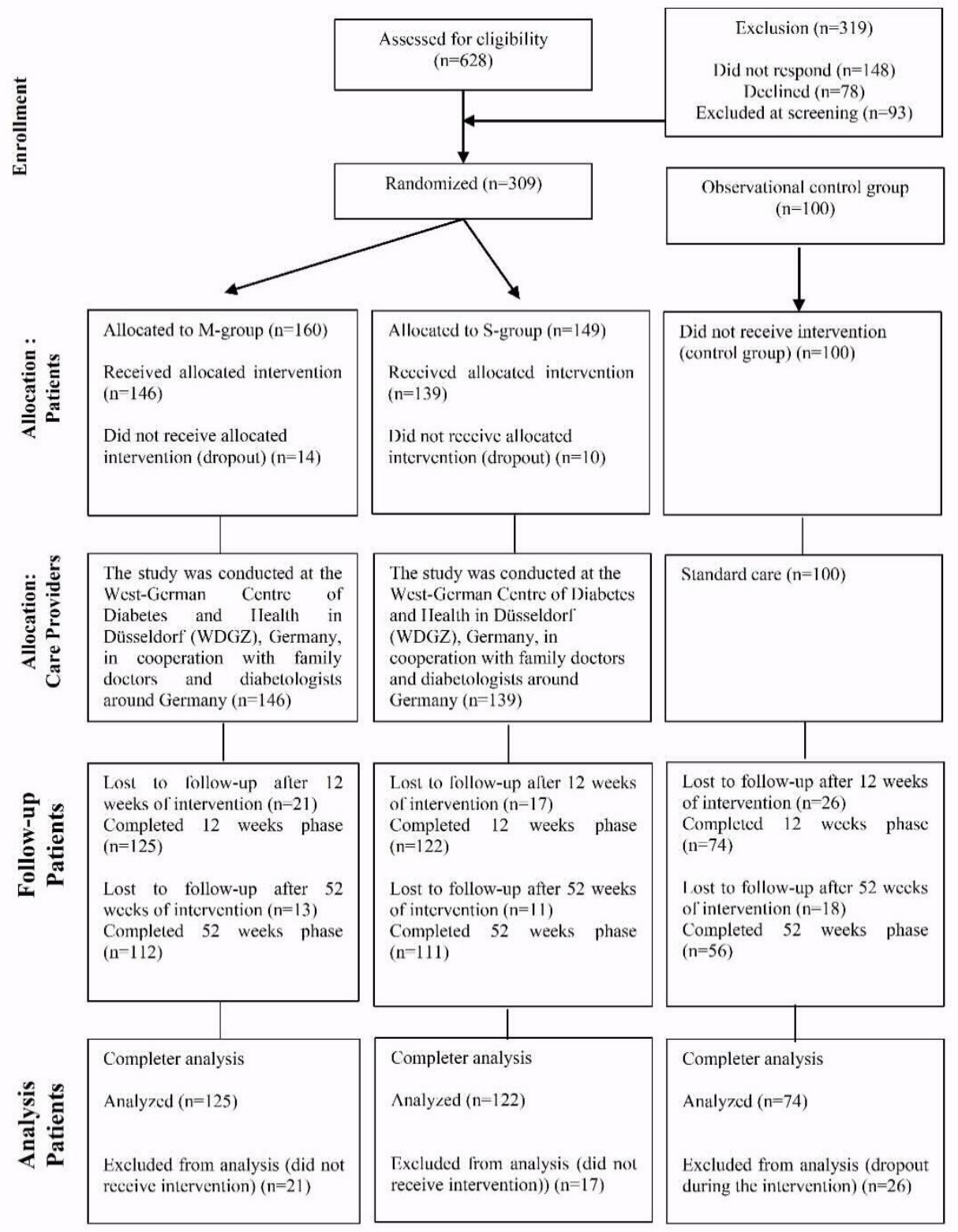

Figure 1. Flow chart.

Besides marginal differences in eating behavior and triglycerides, both intervention groups showed no significant differences in any parameter at week 12 or 52 (Table 2). The ETD in HbA1c reduction after 12 weeks between both intervention groups was $-0.10 \%$ with $95 \% \mathrm{CI}$ [ $-0.40 ; 0.21]$ $(p>0.05)$. Treatment superiority of the S-group vs. M-Group is not statistically significant after the 52-weeks follow-up with $-0.22 \%[-0.56 ; 0.10](p=0.15)$. Furthermore, the proportion of weight loss between both of the intervention groups was not different from baseline to week 12 and week 52 (Figure 2). 
Table 2. Group comparison between S-group and M-group after 12 and 52 weeks (primary endpoints).

\begin{tabular}{|c|c|c|c|c|c|c|}
\hline & \multicolumn{3}{|c|}{12 Weeks } & \multicolumn{3}{|c|}{52 Weeks } \\
\hline Sex (\% male) & 52.5 & 46.4 & 0.374 & 50.4 & 46.3 & 0.593 \\
\hline Weight (kg) & $103 \pm 22$ & $103 \pm 23$ & 0.333 & $98 \pm 17$ & $101 \pm 23$ & 0.245 \\
\hline BMI $\left(\mathrm{kg} / \mathrm{m}^{2}\right)$ & $33.9 \pm 5.6$ & $35.1 \pm 7.5$ & 0.108 & $33.2 \pm 5.1$ & $34.8 \pm 7.6$ & 0.074 \\
\hline $\begin{array}{l}\text { Known diabetes } \\
\text { duration (years) }\end{array}$ & $7.7 \pm 6.6$ & $8.6 \pm 6.4$ & 0.265 & $7.3 \pm 5.2$ & $8.9 \pm 6.6$ & 0.053 \\
\hline $\mathrm{FBG}(\mathrm{mg} / \mathrm{dL})$ & $154 \pm 54$ & $157 \pm 50$ & 0.673 & $156 \pm 51$ & $165 \pm 52$ & 0.163 \\
\hline RR [syst] (mmHg) & $128 \pm 14$ & $129 \pm 16$ & 0.404 & $128 \pm 14$ & $129 \pm 13$ & 0.507 \\
\hline $\mathrm{LDL}(\mathrm{mg} / \mathrm{dL})$ & $116 \pm 36$ & $112 \pm 31$ & 0.357 & $120 \pm 37$ & $111 \pm 33$ & 0.054 \\
\hline Triglyceride (mg/dL) & $193 \pm 111$ & $205 \pm 193$ & 0.564 & $190 \pm 102$ & $368 \pm 534$ & 0.025 \\
\hline $\begin{array}{c}\text { TFEQ [cognitive control] } \\
(\mathrm{au})\end{array}$ & $13(9 ; 16)$ & $13(9 ; 16)$ & 0.590 & $13(9 ; 16)$ & $13(9 ; 16)$ & 0.704 \\
\hline TFEQ [suggestibility] (au) & $5(3 ; 8)$ & $6(3 ; 10)$ & 0.313 & $6(4 ; 8)$ & $6(4 ; 9)$ & 0.189 \\
\hline TFEQ [hunger] (au) & $3(2 ; 6)$ & $4(2 ; 8)$ & 0.131 & $3(1 ; 6)$ & $5(2 ; 8)$ & 0.034 \\
\hline SF36 [physical health] (au) & $46(38 ; 53)$ & $46(35 ; 52)$ & 0.277 & $46(37 ; 52)$ & $42(34 ; 52)$ & 0.052 \\
\hline SF36 [mental health] (au) & $51(35 ; 58)$ & $52(38 ; 58)$ & 0.330 & $49(29 ; 56)$ & $52(37 ; 58)$ & 0.074 \\
\hline No medication (\%) & 8.2 & 6.4 & 0.632 & 8.2 & 4.5 & 0.285 \\
\hline Glucosidase inhibitors (\%) & 0 & 0 & NA & 0.9 & 0 & 0.990 \\
\hline GLP-1 receptor agonists $(\%)$ & 9.0 & 11.2 & 0.674 & 9.0 & 11.6 & 0.661 \\
\hline $\begin{array}{c}\text { Sodium-glucose } \\
\text { co-transporter-2 (\%) }\end{array}$ & 0.8 & 0.8 & 0.990 & 0.9 & 0.9 & 0.990 \\
\hline Insulin (\%) & 18.9 & 13.6 & 0.302 & 18.9 & 15.3 & 0.140 \\
\hline
\end{tabular}

Shown are means \pm standard deviations, median (1st; 3rd quartiles) or percentages. Differences after 12 and 52 weeks between groups were analyzed using multivariable regression models adjusting for baseline values; au, arbitrary units; FBG, fasting blood glucose; BMI, body mass index; DBP, diastolic blood pressure; HDL, high-density-lipoprotein; LDL, low-density-lipoprotein; NA, not applicable; SBP, systolic blood pressure; SF36, short form-36; TFEQ, three-factor eating questionnaire; DDP4, dipeptidyl peptidase 4; GLP-1, glucagon-like peptide-1. 


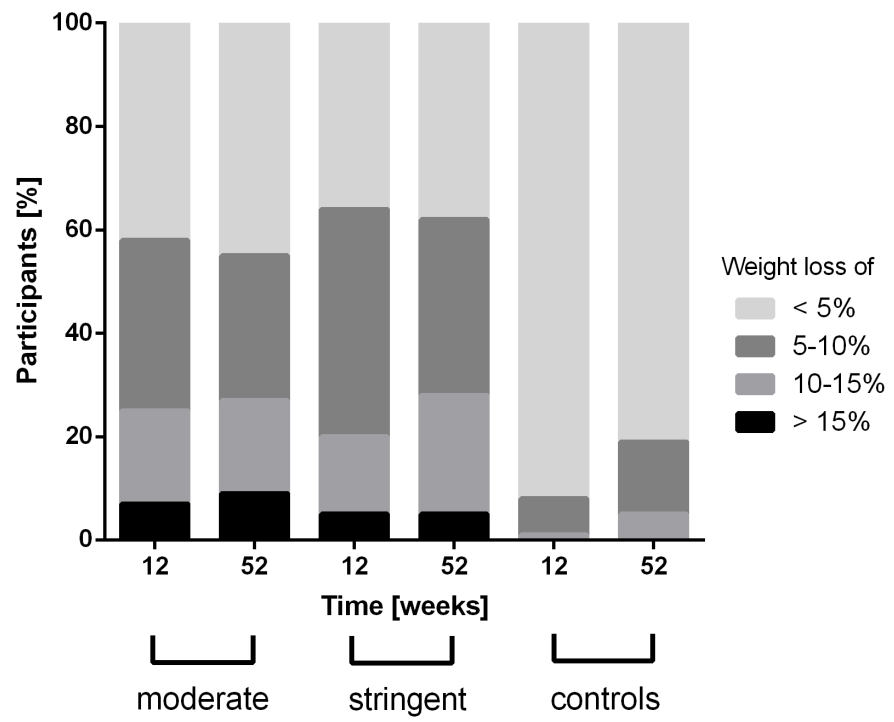

Figure 2. Weight change achieved after 12 and 52 weeks of intervention. Analyses of difference in frequency distribution of weight loss were calculated by using Fisher's exact test.

After 12 weeks of intervention, HbA1c was reduced by (mean [95\% confidence interval (CI)] $-0.97 \%$ [ -1.21 to -0.74$]$ in the S-group and by $-0.84 \%$ [ -1.08 to -0.61$]$ in the M-group (both $p<0.001$ ) as shown in Table 3. These improvements were still significant after the Bonferroni correction for multiple testing. After 52 weeks of follow-up, the reduction of $\mathrm{HbA1c}$ lost its clinical relevance $(\geq 0.60 \%)$ [18] in the M-group with $-0.55 \%$ [ -0.80 to -0.29$]$ when compared to the S-Group with $-0.81 \%$ [ -1.06 to -0.55$]$. Patients of the control group showed no improvement in HbA1c neither after 12 weeks nor after 52 weeks.

Changes of anthropometric, clinical, pharmaceutical, and behavioral parameters within both of the intervention groups after 12 and 52 weeks of intervention are shown in Table 3. Improvements in body weight, BMI, fasting blood glucose, systolic and diastolic blood pressure, as well as eating behavior were observed in the M- and S-group after 12 and 52 weeks of follow-up (all $p<0.01$ ). These changes in $\mathrm{HbA1c}$, weight, BMI, fasting blood glucose, systolic and diastolic blood pressure, as well as eating behavior were still significant after the Bonferroni correction for multiple testing $(p$ value $=0.002)$ in the within-groups analysis. Doses of anti-diabetic medication was already adjusted within the first week of intervention. Frequencies of anti-diabetic drugs were not significantly changed within groups after Bonferroni correction.

Table 3. Changes of anthropometric, clinical, pharmaceutical, and behavioral parameters (secondary endpoints).

\begin{tabular}{|c|c|c|c|}
\hline & M-Group ( $n=125)$ & S-Group $(n=122)$ & $p$ \\
\hline $\mathrm{HbA1c}(\%)$ & $8.4 \pm 1.1$ & $8.4 \pm 1.2$ & \\
\hline$\Delta \mathrm{HbA} 1 \mathrm{c}(\%) 12$ weeks & $-0.84[-1.08 ;-0.61]^{* * *, \mathrm{a}}$ & $-0.97[-1.21 ;-0.74]^{* * *, \mathrm{a}}$ & 0.538 \\
\hline$\Delta \mathrm{HbA} 1 \mathrm{c}(\%) 52$ weeks & $-0.55[-0.80 ;-0.29]^{* * *, \mathrm{a}}$ & $-0.81[-1.06 ;-0.55]^{* * *, \mathrm{a}}$ & 0.149 \\
\hline Weight $(\mathrm{kg})$ & $110 \pm 24$ & $107 \pm 20$ & \\
\hline$\Delta$ Weight (kg) 12 weeks & $-6.93[-8.08 ;-5.78]^{* * *, \mathrm{a}}$ & $-6.91[-8.07 ;-5.76]^{* * *, \mathrm{a}}$ & 0.999 \\
\hline$\Delta$ Weight $(\mathrm{kg}) 52$ weeks & $-7.30[-8.65 ;-5.95]^{* * *, \mathrm{a}}$ & $-7.45[-8.80 ;-6.10] * * *, \mathrm{a}$ & 0.615 \\
\hline BMI $\left(\mathrm{kg} / \mathrm{m}^{2}\right)$ & $37.5 \pm 7.6$ & $36.1 \pm 5.9$ & \\
\hline$\Delta \mathrm{BMI}\left(\mathrm{kg} / \mathrm{m}^{2}\right) 12$ weeks & $-2.38[-2.78 ;-1.98]^{* * *, \mathrm{a}}$ & $-2.35[-2.75 ;-1.95]^{* * *, \mathrm{a}}$ & 0.911 \\
\hline$\Delta \mathrm{BMI}\left(\mathrm{kg} / \mathrm{m}^{2}\right) 52$ weeks & $-2.36[-2.84 ;-1.88]^{* * *, \mathrm{a}}$ & $-2.50[-2.98 ;-2.02]^{* * *, \mathrm{a}}$ & 0.536 \\
\hline $\mathrm{FBG}(\mathrm{mg} / \mathrm{dL})$ & $181 \pm 53$ & $178 \pm 63$ & \\
\hline$\Delta \mathrm{FBG}(\mathrm{mg} / \mathrm{dL}) 12$ weeks & $-24[-34 ;-13]^{* * *, \mathrm{a}}$ & $-25[-36 ;-15]^{* * *, \mathrm{a}}$ & 0.791 \\
\hline$\Delta \mathrm{FBG}(\mathrm{mg} / \mathrm{dL}) 52$ weeks & $-17[-30 ;-5]^{* *}$ & $-22[-35 ;-10]^{* * *, a}$ & 0.196 \\
\hline $\mathrm{SBP}(\mathrm{mmHg})$ & $136 \pm 17$ & $134 \pm 14$ & \\
\hline
\end{tabular}


Table 3. Cont

\begin{tabular}{|c|c|c|c|}
\hline & M-Group $(n=125)$ & S-Group $(n=122)$ & $p$ \\
\hline$\Delta \mathrm{SBP}(\mathrm{mmHg}) 12$ weeks & $-5.6[-8.7 ;-2.5]^{* * *, \mathrm{a}}$ & $-6.6[-9.7 ;-3.5]^{* * *, \mathrm{a}}$ & 0.512 \\
\hline$\triangle \mathrm{SBP}(\mathrm{mmHg}) 52$ weeks & $-6.0[-9.3 ;-2.7] * * *, \mathrm{a}$ & $-5.8[-9.1 ;-2.5]^{* * *, \mathrm{a}}$ & 0.858 \\
\hline $\mathrm{DBP}(\mathrm{mmHg})$ & $82 \pm 8$ & $80 \pm 8$ & \\
\hline$\Delta \mathrm{DBP}(\mathrm{mmHg}) 12$ weeks & $-2.9[-4.5 ;-1.3]^{* * *, \mathrm{a}}$ & $-3.0[-4.6 ;-1.4]^{* * *, \mathrm{a}}$ & 0.371 \\
\hline$\Delta \mathrm{DBP}(\mathrm{mmHg}) 52$ weeks & $-3.7[-5.6 ;-1.9] * * *, \mathrm{a}$ & $-2.9[-4.8 ;-1.0]^{* *}$ & 0.992 \\
\hline Total cholesterol (mg/dL) & $200 \pm 52$ & $198 \pm 43$ & \\
\hline$\Delta$ Total cholesterol $(\mathrm{mg} / \mathrm{dL}) 12$ weeks & $-11.1[-18.9 ;-3.3]^{* *}$ & $-7.0[-14.7 ; 0.8]$ & 0.565 \\
\hline$\Delta$ Total cholesterol $(\mathrm{mg} / \mathrm{dL}) 52$ weeks & $-8.0[-17.3 ; 1.3]$ & $0.1[-9.3 ; 9.4]$ & 0.396 \\
\hline $\mathrm{HDL}(\mathrm{mg} / \mathrm{dL})$ & $46 \pm 10$ & $47 \pm 11$ & \\
\hline$\Delta \mathrm{HDL}(\mathrm{mg} / \mathrm{dL}) 12$ weeks & $-0.1[-1.6 ; 1.4]$ & $-0.1[-1.6 ; 1.4]$ & 0.908 \\
\hline$\Delta \mathrm{HDL}(\mathrm{mg} / \mathrm{dL}) 52$ weeks & $0.9[-4.0 ; 5.9]$ & $4.5[-0.5 ; 9.5]$ & 0.248 \\
\hline $\mathrm{LDL}(\mathrm{mg} / \mathrm{dL})$ & $118 \pm 32$ & $119 \pm 37$ & \\
\hline$\Delta \mathrm{LDL}(\mathrm{mg} / \mathrm{dL}) 12$ weeks & $-6.6[-10.9 ;-2.3] * *$ & $-3.3[-7.6 ; 1.0]$ & 0.144 \\
\hline$\Delta \mathrm{LDL}(\mathrm{mg} / \mathrm{dL}) 52$ weeks & $-7.6[-12.8 ;-2.4]^{* *}$ & $1.8[-3.4 ; 7.0]$ & 0.012 \\
\hline Triglyceride $(\mathrm{mg} / \mathrm{dL})$ & $383 \pm 586$ & $220 \pm 157$ & \\
\hline$\Delta$ Triglyceride $(\mathrm{mg} / \mathrm{dL}) 12$ weeks & $-186[-268 ;-104]^{* * *, a}$ & $-27[-109 ; 56]$ & 0.041 \\
\hline$\Delta$ Triglyceride (mg/dL) 52 weeks & $-35[-86 ; 17]$ & $-31[-83 ; 21]$ & 0.865 \\
\hline TFEQ [cognitive control] (au) & $9.7 \pm 3.9$ & $10.0 \pm 4.3$ & \\
\hline$\Delta$ TFEQ [cognitive control] (au) 12 weeks & $2.5[1.7 ; 3.3] * * *, \mathrm{a}$ & $2.5[1.7 ; 3.3] * * *, \mathrm{a}$ & 0.847 \\
\hline$\Delta$ TFEQ [cognitive control] (au) 52 weeks & $2.3[1.5 ; 3.1] * * *, \mathrm{a}$ & $2.2[1.4 ; 3.0] * * *, \mathrm{a}$ & 0.633 \\
\hline TFEQ [suggestibility] (au) & $7.4 \pm 3.8$ & $7.0 \pm 3.5$ & \\
\hline$\Delta$ TFEQ [suggestibility] (au) 12 weeks & $-0.8[-1.3 ;-0.3]^{* *}$ & $-0.8[-1.4 ;-0.3]^{* * *, \mathrm{a}}$ & 0.686 \\
\hline$\Delta$ TFEQ [suggestibility] (au) 52 weeks & $-0.8[-1.3 ;-0.2]^{* *}$ & $-0.9[-1.4 ;-0.4]^{* * *, \mathrm{a}}$ & 0.342 \\
\hline TFEQ [hunger] (au) & $6.3 \pm 3.7$ & $5.6 \pm 3.3$ & \\
\hline$\Delta$ TFEQ [hunger] (au) 12 weeks & $-1.3[-1.8 ;-0.7]^{* * *, \mathrm{a}}$ & $-1.3[-1.9 ;-0.7]]^{* * * a}$ & 0.586 \\
\hline$\Delta$ TFEQ [hunger] (au) 52 weeks & $-1.1[-1.7 ;-0.5] * * *, \mathrm{a}$ & $-1.4[-2.0 ;-0.8]^{* * *, \mathrm{a}}$ & 0.074 \\
\hline SF36 [physical health] (au) & $42 \pm 10$ & $43 \pm 10$ & \\
\hline$\Delta$ SF36 [physical health] (au) 12 weeks & $1.5[-0.2 ; 3.2]$ & $1.4[-0.3 ; 3.1]$ & 0.773 \\
\hline$\Delta$ SF36 [physical health] (au) 52 weeks & $0.2[1.4 ; 1.8]$ & $1.2[-0.4 ; 2.8]$ & 0.150 \\
\hline SF36 [mental health] (au) & $47 \pm 13$ & $45 \pm 15$ & \\
\hline$\Delta$ SF36 [mental health] (au) 12 weeks & $0.6[-2.0 ; 3.2]$ & $1.2[-1.5 ; 3.8]$ & 0.953 \\
\hline$\Delta$ SF36 [mental health] (au) 52 weeks & $-0.4[-3.0 ; 2.2]$ & $-1.4[-3.9 ; 1.2]$ & 0.272 \\
\hline No medication $(\%)$ & 8.0 & 8.2 & \\
\hline$\Delta$ no medication $(\%) 12$ weeks & -1.6 & 0 & 0.652 \\
\hline$\Delta$ no medication $(\%) 52$ weeks & -3.5 & -0.1 & 0.179 \\
\hline Metformin $(\%)$ & 81.6 & 77.0 & \\
\hline$\Delta$ Metformin $(\%) 12$ weeks & 0 & -0.8 & 0.660 \\
\hline$\Delta$ Metformin (\%) 52 weeks & -1.2 & 0.5 & 0.942 \\
\hline DPP4 inhibitors $(\%)$ & 28.8 & 24.6 & \\
\hline$\Delta$ DPP4 inhibitors (\%) 12 weeks & 0.8 & -0.8 & 0.314 \\
\hline$\Delta$ DPP4 inhibitors (\%) 52 weeks & 5.1 & 0.6 & 0.377 \\
\hline Sulfonylurea (\%) & 6.4 & 4.1 & \\
\hline$\Delta$ Sulfonylurea $(\%) 12$ weeks & -2.4 & -2.5 & 1.000 \\
\hline$\Delta$ Sulfonylurea (\%) 52 weeks & 2.5 & 0.4 & 0.920 \\
\hline Glinides (\%) & 0 & 0 & \\
\hline$\Delta$ Glinides (\%) 12 weeks & 0 & 0 & NA \\
\hline$\Delta$ Glinides (\%) 52 weeks & 2.7 & 0.9 & 0.622 \\
\hline Glitazone (\%) & 1.6 & 0.8 & \\
\hline$\Delta$ Glitazone $(\%) 12$ weeks & -1.6 & 0 & 0.428 \\
\hline$\Delta$ Glitazone (\%) 52 weeks & -0.5 & -0.8 & 1.000 \\
\hline Glucosidase inhibitors (\%) & 0 & 0.8 & \\
\hline$\Delta$ Glucosidase inhibitors (\%) 12 weeks & 0 & 0 & NA \\
\hline$\Delta$ Glucosidase inhibitors (\%) 52 weeks & 0 & 0.1 & 1.000 \\
\hline GLP -1 receptor agonists $(\%)$ & 12.0 & 8.2 & \\
\hline$\Delta \mathrm{GLP}-1$ receptor agonists (\%) 12 weeks & -0.8 & 0.8 & 0.855 \\
\hline$\Delta \mathrm{GLP}-1$ receptor agonists (\%) 52 weeks & 0.4 & 0.8 & 1.000 \\
\hline Sodium-glucose co-transporter-2 (\%) & 0.8 & 0.8 & \\
\hline$\Delta$ Sodium-glucose co-transporter-2 (\%) 12 weeks & 0 & 0 & NA \\
\hline$\Delta$ Sodium-glucose co-transporter-2 (\%) 52 weeks & 0.1 & 0.1 & 1.000 \\
\hline Insulin (\%) & 19.2 & 19.7 & \\
\hline$\Delta$ Insulin (\%) 12 weeks & -5.6 & -0.8 & 0.290 \\
\hline$\Delta$ Insulin (\%) 52 weeks & -3.9 & -0.8 & 0.256 \\
\hline
\end{tabular}

Data are shown as mean \pm SD and mean $[95 \% \mathrm{CI}]$ or $\%$ as appropriate; ${ }^{* *} p<0.001$ vs. baseline; ${ }^{* *} p<0.01$ vs. baseline; Superscript letter a represents significance after Bonferroni correction for multiple testing $(p<0.002)$. Differences in changes after 12 and 52 weeks between both groups were analyzed using multivariable regression models adjusting baseline values. au, arbitrary units; BMI, body mass index; DBP, diastolic blood pressure; SBP, systolic blood pressure; SF36, short form-36 questionnaire; FBG, fasting blood glucose; HDL, high-density-lipoprotein; LDL, low-density-lipoprotein; TFEQ, three-factor eating questionnaire; DDP4, dipeptidyl peptidase 4; GLP-1, glucagon-like peptide-1. NA, not applicable. 
When compared to the control group (12 weeks: $-0.20 \pm 0.80$ standard deviation (SD); 52 weeks: $-0.10 \pm 0.90 \mathrm{SD})$, only the S-group (12 weeks: $-0.97 \pm 1.18$ SD; 52 weeks: $-0.81 \pm 1.20 \mathrm{SD})$ demonstrated a significant difference in HbA1c after 52 weeks of follow-up $(p<0.01)$, while the M-group (-0.84 \pm 1.14 SD; 52 weeks: $-0.55 \pm 1.31$ SD) was not significantly different (Figure 3). Furthermore, a higher proportion of participants with a larger weight reduction was shown in the intervention groups after 12 and 52 weeks in comparison to the control group (all $p<0.001$; Figure 2).

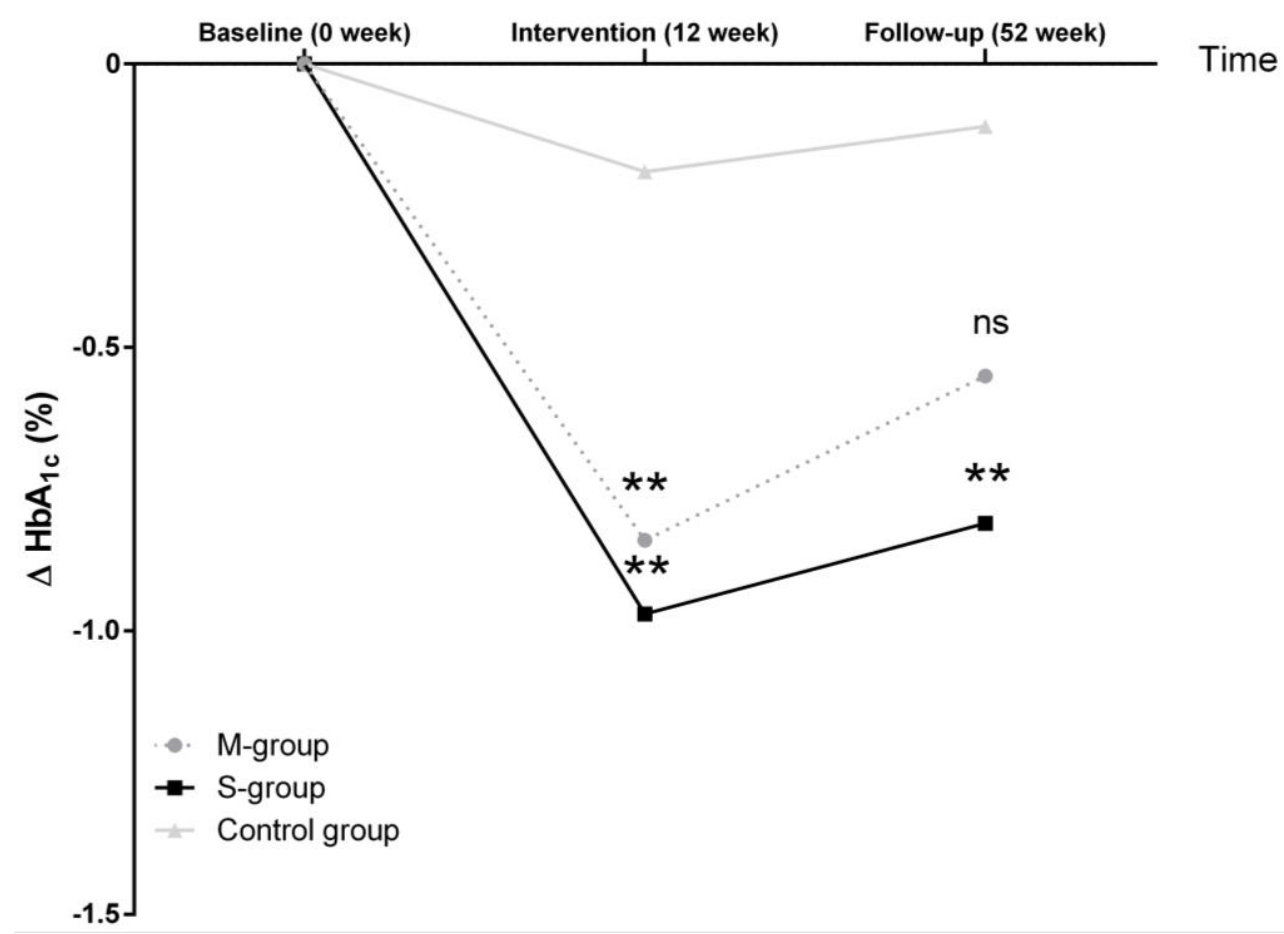

Figure 3. Change of glycemic control after 12 weeks of intervention and 52 weeks of follow-up. At baseline, M-, S- and control group were not significantly different, however, 12 weeks of diet intervention led to reductions in $\mathrm{HbA} 1 \mathrm{c}$ in both intervention groups in comparison to the control group. Compared to controls, only the S-group showed a significant difference in $\mathrm{HbA1c}$ after 52 weeks of follow-up. Analyses of variance with repeated measures were performed to determine differences between groups; ns, not significant; ${ }^{* *} p<0.01$ vs. controls.

\section{Discussion}

The results of the present study demonstrate that an individualized meal replacement therapy starting with intense low-carbohydrate formula diets and SMBG-accompanied reintervention of low-carbohydrate meals lead to clinically relevant improvements in HbA1c after 12 weeks of intervention in patients with poorly controlled long-standing type 2 diabetes. Particularly, patients of the more intense intervention group (S-group) showed long-term clinically relevant improvements after 52 weeks of follow-up as compared to the participants of the moderate intervention group (M-group), although this difference was not statistically significant. Furthermore, the overall dropout rate after allocation into both intervention groups was small $(247: 285 ; 13 \%)$ and not different (S-group $=12 \%$ and $\mathrm{M}$-group $=14 \%$ ). We hypothesize that the strict rules, the stringent and individual SMBG [17], and the complete replacement of all meals in the S-group during the first week contributed to a subtler change of behavior and higher motivation for the diet, which was shown to be necessary for long-term changes of behavior in high-risk individuals for type 2 diabetes in prior studies [19]. Furthermore, we assume that our personalized nutrition and treatment-approach with a more intense patient empowerment during the first week in the S-group contributed to a long-term difference in HbA1c after 52 weeks of follow-up. The recently published DIRECT study has demonstrated that 
a strict calorie restriction with only $825-853 \mathrm{kcal}$ per day for 3-5 months contributes to significant improvements of $\mathrm{HbA} 1 \mathrm{c}(-0.9 \%)$ and body weight, and in the further course to diabetes remission after 52 weeks of intervention [13]. However, the formula diet contained proteins and carbohydrates in a ratio of 1:2 [13]. We chose an opposite formula diet that was high in protein, but low in carbohydrates (ratio nearly 2:1) with individualized moderate-calorie supply (1300-1500 kcal/day), because we postulate that a higher amount of carbohydrates would stimulate an increase in insulin release and a decrease in fat burning [20]. Therefore, our strong carbohydrate reduction with an accompanied stepped food reintroduction should lead to long-term benefits like it was shown before in the DIRECT study [13]. Another explanation could be that the S-group was somewhat higher motivated to be physically active due to the complete change of nutrition and behavior, respectively. Previous studies have already demonstrated strong effects on $\mathrm{HbA1c}$ through very low-calorie liquid formula diets in small groups of patients with type 2 diabetes $(n<30)$ during an investigation period up to 26 weeks, especially after a short duration of diabetes (<4 years) $[11,14,15]$. This correlation between diabetes duration and changes of $\mathrm{HbA} 1 \mathrm{c}$ after 12 or 52 weeks could be confirmed in our study by the whole intervention cohort ( $r=0.226$ (after 12 weeks) or 0.229 (after 52 weeks); both $p<0.001$ ), independently of age.

Our approach of low-carbohydrate meal replacement is based on the recommendations for diets in type 2 diabetes, as well as recently published reviews and meta-analyses [21,22]. Although, a healthy diet is crucial for type 2 diabetes, there still exists controversy in the field about the feasibility and mechanisms of these stringent types of dietary interventions and their long-term effects in $\mathrm{HbA1c}$ [23]. The effects on the glucose metabolism (e.g., anti-diabetic medication was adjusted within the first week) occur immediately after beginning the meal replacement therapy [12] and before a significant weight loss takes place. The observed effects are comparable with those after bariatric surgery [7]. Possible explanation approaches in this context could be altered levels of incretin secretion [24], improved mitochondrial oxidative function [25], energy restriction [10], the sudden negative energy balance [14], or a combination of all these points. Furthermore, a reduced carbohydrate intake [26] or a reduced number of carbohydrate-containing meals might trigger the fast effects on the glucose metabolism. This would be in line with observations that two meals per day are better than six [27] for type 2 diabetes patients, especially in terms of body weight, insulin resistance/sensitivity, and beta cell function [10]. The results of the PREDIMED study, in which two high-fat/lower-carbohydrate Mediterranean diets were compared to a fat-reduced diet regarding the incidence of type 2 diabetes [28] or cardiovascular events [29], as well as changes of body weight and waist circumference [30], support our findings that carbohydrate-reduced diets are beneficial for patients with type 2 diabetes.

The improvements in glycemic control in both intervention groups in the present study were followed by strong reductions in body weight (Figure 2). In a recently published meta-analysis, it was shown that very low-calorie ( $<800 \mathrm{kcal}$ per day) or low-energy liquid-formula ( $>800 \mathrm{kcal}$ per day) diets can induce large reductions of body weight (ranging from 8.9 to $15.0 \mathrm{~kg}$ ) in obese people (BMI: $35.5-42.6 \mathrm{~kg} / \mathrm{m}^{2}$ ) with and without type 2 diabetes [31]. The slight difference in body weight reduction in our trial can be explained by a higher calorie consumption per day ( $\approx 1300-1500$ kcal per day) when compared to the studies of the meta-analysis. Furthermore, our results are comparable to the findings of Steven et al. [11], who works with a very low-calorie and moderate-carbohydrate composition (43\% carbohydrate, 34\% protein, and 19.5\% fat; $2.6 \mathrm{MJ} /$ day [624 kcal/day]). They found that a very low-calorie diet over eight weeks can contribute to a meaningful weight reduction of $\approx 14 \mathrm{~kg}$, which was still comparably high, even after 26 weeks $(\approx 13 \mathrm{~kg})$ in individuals with type 2 diabetes. In regard to the aforementioned findings, we could demonstrate similar results of weight reduction with $\approx 7 \mathrm{~kg}$ after 12 and 52 weeks of intervention. In contrast to Steven et al., we designed an individualized low-carbohydrate and moderate-calorie diet intervention (31\% carbohydrate), accompanied with SMBG as it might be more feasible for patients with type 2 diabetes, characterized with eating and motivation impairments [32]. The improvements, apart from the meal restriction, could be therefore also explained by improved education regarding nutrition, physical activity, and blood glucose control. 
A recently published review supports our approach, as it states that a rather moderate weight loss is more sufficient for the transition from metabolically unhealthy obesity to metabolically healthy obesity with a lower risk for adverse outcomes in the long run than a large amount of weight loss in a short period [33]. We chose this calorie goal per day in order to reduce the rate for dropouts and increase the participants therapy adherence. Lifestyle interventions are always criticized in terms of their long-term effectiveness, and one possible hypothesis says that the major problem is that patients fail to adhere to the altered lifestyle prescriptions [34]. In contrast to many other long-term lifestyle intervention programs [35], the relatively high number of completers after $12(83 \%)$ and $52(72 \%)$ weeks supports our study design and approach. Potential reasons for nonadherence comprise: age, perception and duration of disease, polytherapy, social and psychological factors, costs, dislike for foods included in meal plans, education and a lack of understanding of the long-term benefits of treatment, adverse outcomes (e.g., weight gain or hypoglycemia), as well as negative treatment perceptions [36]. In this context, new innovative methods are needed to assist those patients. In light of these problems, we designed the study with almost no barriers for the participants (e.g., 1:1 personal support or no additional costs) and provided every participant with a personalized meal replacement and supported them in their SMBG.

Further improvements were achieved in the cardiometabolic parameters of fasting blood glucose, as well as systolic and diastolic blood pressure. These results are confirmative regarding other studies with low-calorie diets in patients with a short- and long-duration type 2 diabetes and moderate [14] or poor glycemic control $[10,15]$. Our results are also confirmed by a recently published review in terms of improvements of the cardiovascular risk profile in patients with type 2 diabetes showing a significant decrease in systolic and diastolic blood pressure as well as fasting blood glucose after low-calorie diets [37].

A further positive effect following the intervention was the improvement of eating behavior in the intervention groups. The simple and structured formula diet reduced feelings of hunger and increased the control regarding eating-associated actions. In patients with type 2 diabetes, a disordered eating behavior can be present and it is associated with poor quality of life [38]. When compared to individuals with the metabolic syndrome, type 2 diabetes participants of the present intervention groups showed a pronounced feeling of hunger and a weaker control over their suggestibility for food [39]. Another study supports our findings showing meaningful improvements in eating behavior after a three-month mindful eating intervention in non-insulin requiring patients with type 2 diabetes in a small cohort $(n<30)$ [40].

A previously published pilot study [12] revealed how a formula diet affects blood glucose control and weight, and how insulin is reduced or discontinued. However, it was also shown that participants sometimes found it difficult to maintain the stringent diet during the first week. Therefore, we were interested in whether a moderate approach also leads to success. The underlying idea was that the replacement of all three meals in the first week would lead to some kind of "reset". In combination with concomitant blood glucose self-monitoring in the following weeks, an individualized diet should be gradually rebuilt. However, because of the similarity of the intervention design, we expected that the moderate diet regimen would lead to a significant improvement as well. We, therefore, included the comparison with a control group that received standard treatment.

The strengths of the present study comprise: (i) a relatively large number of patients studied per group who had poor controlled type 2 diabetes and a long type 2 diabetes duration; (ii) a longer study period compared to previous studies with formula diets (52 weeks vs. $\leq 26$ weeks); as well as (iii) a randomized trial design with two intervention groups and one observational control group. Furthermore, the (iv) chosen real-world setting with a combination of formula diet, SMBG, and dietary education could be easily implemented into present health care programs. Likewise, another study with a real-world approach could demonstrate that even the partial use of a formula diet with one pack of formula diet instead of one of three daily low-caloric meals for 24 weeks was much more effective in 
reducing body weight and improving coronary risk factors than a conventional diet with a reduced energy intake in obese type 2 diabetic patients [41].

A limitation of our study is that we did not use food diaries to control for decreased calorie consumption or incorrect food compositions (e.g., the amount of carbohydrate in the diet, glycemic index, fat or protein intake) after the acute meal replacement phase from week 13 to week 52 . However, the 52-week follow-up revealed that participants of both intervention groups showed no difference in maintaining the formula diet and following the dietary intervention until the study end (S-group $65 \%$; M-group 63\%). Also, more profound and quantitative diagnostics, such as isotope measurements, could have been done to control for food-related study compliance. On the other side, interventional studies with formula diets and similar results in a real-world setting [31] support our therapeutic approach in patients with poorly controlled type 2 diabetes.

Another factor, which should be considered, is the adjusted glucose-lowering medication dose in response to glycemic improvements due to the meal replacement intervention. It is conceivable that the impact of our formula diet on the $\mathrm{HbA} 1 \mathrm{c}$ reduction is underestimated due to this adjustment. Another limitation of our real-world study is that the participants of the control group were not randomly assigned. In one of our previously published studies (NCT02066831), we found dramatic negative effects on $\mathrm{HbA} 1 \mathrm{c}$ and dropout rate (26\%) for the participants of the control group [16]. This approach, without a randomized control group with standard care, was also conducted in other benchmark studies for formula diet trials, like the Counterbalance Study (CS) [14] and the Counterpoint Study (CP) [15]. Both of the studies with small sample sizes $(n=11-29)$ reduced HbA1c (CS: $-1.4 \%$ and $\mathrm{CP}:-1.1 \%$ to $-0.6 \%$ ) similar, as it was shown in our study after eight weeks of intervention. Furthermore, when comparing the present study results with findings from other landmark studies (DIRECT and TeLiPro study $[17,21]$ ), one can see that an assignment to the control group with standard care is accompanied with serious and disadvantageous effects, such as high dropout rates or even an increase in $\mathrm{HbA} 1 \mathrm{c}$. These findings support our approach and study design.

In sum, individualized low-carbohydrate diets can produce clinically-relevant reductions in $\mathrm{HbA} 1 \mathrm{c}$ after 12 weeks of intervention. Furthermore, body weight, fasting blood glucose, quality of life, eating behavior, and other cardiometabolic risk factors improved, although not all of the parameters showed statistically significant improvements. Moreover, the initiation of a weight loss program with one week of full meal replacement (three meals per day) resulted in a clinically relevant long-term $\mathrm{HbA} 1 \mathrm{c}$ reduction, when compared to an observational control group that had standard care. Our practicable and real-world setting-based approach led to relevant long-term improvements that were comparable with procedures of bariatric surgery without adverse events or negative side-effects. These results support the therapeutic concept of low-carbohydrate diets by formula diets in patients with poorly controlled type 2 diabetes.

Supplementary Materials: The following are available online at http:/ /www.mdpi.com/2072-6643/10/8/1022/ s1. Table S1. Baseline characteristics of the drop-outs. Table S2. Baseline antidiabetic drugs.

Author Contributions: M.R. and K.K. wrote the manuscript, researched and collected data. M.R. and K.K. performed the statistical analysis. S.M. had the idea, initiated the study and revised the manuscript. K.N. and B.G. researched data and contributed to the manuscript. S.M. is the guarantor of this work and, as such, had full access to all the data in the study and take responsibility for the integrity of the data and the accuracy of the data analysis.

Funding: The study was financially supported by the Almased-Wellness-GmbH and the Gesellschaft von Freunden und Förderern der Heinrich-Heine-Universität Düsseldorf e.V. The funder had no influence on study design, data collection, data analysis, manuscript preparation and/or publication decisions.

Acknowledgments: We thank our study nurse Bettina Prete (West-German Centre of Diabetes and Health) for her excellent work.

Conflicts of Interest: K. Kempf and S. Martin received consultant fees from the Almased-Wellness-GmbH. M. Röhling, B. Gärtner and K. Niedermeier declare that there is no conflict of interest regarding the publication of this article. 


\section{References}

1. Dunkley, A.J.; Bodicoat, D.H.; Greaves, C.J.; Russell, C.; Yates, T.; Davies, M.J.; Khunti, K. Diabetes prevention in the real world: Effectiveness of pragmatic lifestyle interventions for the prevention of type 2 diabetes and of the impact of adherence to guideline recommendations: A systematic review and meta-analysis. Diabetes Care 2014, 37, 922-933. [CrossRef] [PubMed]

2. Rise, M.B.; Pellerud, A.; Rygg, L.O.; Steinsbekk, A. Making and maintaining lifestyle changes after participating in group based type 2 diabetes self-management educations: A qualitative study. PLoS ONE 2013, 8, e64009. [CrossRef] [PubMed]

3. Giorgino, F.; Home, P.D.; Tuomilehto, J. Glucose Control and Vascular Outcomes in Type 2 Diabetes: Is the Picture Clear? Diabetes Care 2016, 39 (Suppl. S2), S187-S195. [CrossRef] [PubMed]

4. U.K. Prospective Diabetes Study Group. U.K. prospective diabetes study 16. Overview of 6 years' therapy of type II diabetes: A progressive disease. U.K. Prospective Diabetes Study Group. Diabetes 1995, 44, 1249-1258. [CrossRef]

5. Yki-Jarvinen, H.; Ryysy, L.; Kauppila, M.; Kujansuu, E.; Lahti, J.; Marjanen, T.; Niskanen, L.; Rajala, S.; Salo, S.; Seppala, P.; et al. Effect of obesity on the response to insulin therapy in noninsulin-dependent diabetes mellitus. J. Clin. Endocrinol. Metab. 1997, 82, 4037-4043. [CrossRef] [PubMed]

6. Gloy, V.L.; Briel, M.; Bhatt, D.L.; Kashyap, S.R.; Schauer, P.R.; Mingrone, G.; Bucher, H.C.; Nordmann, A.J. Bariatric surgery versus non-surgical treatment for obesity: A systematic review and meta-analysis of randomised controlled trials. BMJ 2013, 347, f5934. [CrossRef] [PubMed]

7. Ackerman, N.B. Observations on the improvements in carbohydrate metabolism in diabetic and other morbidly obese patients after jejunoileal bypass. Surg. Gynecol. Obstet. 1981, 152, 581-586. [PubMed]

8. Backman, O.; Stockeld, D.; Rasmussen, F.; Naslund, E.; Marsk, R. Alcohol and substance abuse, depression and suicide attempts after Roux-en-Y gastric bypass surgery. Br. J. Surg. 2016, 103, 1336-1342. [CrossRef] [PubMed]

9. Colquitt, J.L.; Pickett, K.; Loveman, E.; Frampton, G.K. Surgery for weight loss in adults. Cochrane Database Syst. Rev. 2014, 8, Cd003641. [CrossRef] [PubMed]

10. Jackness, C.; Karmally, W.; Febres, G.; Conwell, I.M.; Ahmed, L.; Bessler, M.; McMahon, D.J.; Korner, J. Very low-calorie diet mimics the early beneficial effect of Roux-en-Y gastric bypass on insulin sensitivity and beta-cell Function in type 2 diabetic patients. Diabetes 2013, 62, 3027-3032. [CrossRef] [PubMed]

11. Steven, S.; Hollingsworth, K.G.; Al-Mrabeh, A.; Avery, L.; Aribisala, B.; Caslake, M.; Taylor, R. Very Low-Calorie Diet and 6 Months of Weight Stability in Type 2 Diabetes: Pathophysiological Changes in Responders and Nonresponders. Diabetes Care 2016, 39, 808-815. [CrossRef] [PubMed]

12. Kempf, K.; Schloot, N.C.; Gartner, B.; Keil, R.; Schadewaldt, P.; Martin, S. Meal replacement reduces insulin requirement, $\mathrm{HbA} 1 \mathrm{c}$ and weight long-term in type 2 diabetes patients with $>100 \mathrm{U}$ insulin per day. J. Hum. Nutr. Diet. 2014, 27 (Suppl. S2), 21-27. [CrossRef] [PubMed]

13. Lean, M.E.; Leslie, W.S.; Barnes, A.C.; Brosnahan, N.; Thom, G.; McCombie, L.; Peters, C.; Zhyzhneuskaya, S.; Al-Mrabeh, A.; Hollingsworth, K.G.; et al. Primary care-led weight management for remission of type 2 diabetes (DiRECT): An open-label, cluster-randomised trial. Lancet 2017. [CrossRef]

14. Lim, E.L.; Hollingsworth, K.G.; Aribisala, B.S.; Chen, M.J.; Mathers, J.C.; Taylor, R. Reversal of type 2 diabetes: Normalisation of beta cell function in association with decreased pancreas and liver triacylglycerol. Diabetologia 2011, 54, 2506-2514. [CrossRef] [PubMed]

15. Steven, S.; Taylor, R. Restoring normoglycaemia by use of a very low calorie diet in long- and short-duration Type 2 diabetes. Diabet. Med. 2015, 32, 1149-1155. [CrossRef] [PubMed]

16. Kempf, K.; Altpeter, B.; Berger, J.; Reuss, O.; Fuchs, M.; Schneider, M.; Gartner, B.; Niedermeier, K.; Martin, S. Efficacy of the Telemedical Lifestyle intervention Program TeLiPro in Advanced Stages of Type 2 Diabetes: A Randomized Controlled Trial. Diabetes Care 2017, 40, 863-871. [CrossRef] [PubMed]

17. Kempf, K.; Kruse, J.; Martin, S. ROSSO-in-praxi follow-up: Long-term effects of self-monitoring of blood glucose on weight, hemoglobin A1c, and quality of life in patients with type 2 diabetes mellitus. Diabetes Technol. Ther. 2012, 14, 59-64. [CrossRef] [PubMed]

18. Thomas, D.E.; Elliott, E.J.; Naughton, G.A. Exercise for type 2 diabetes mellitus. Cochrane Database Syst. Rev. 2006, 3, Cd002968. [CrossRef] [PubMed] 
19. den Braver, N.R.; de Vet, E.; Duijzer, G.; Ter Beek, J.; Jansen, S.C.; Hiddink, G.J.; Feskens, E.J.M.; Haveman-Nies, A. Determinants of lifestyle behavior change to prevent type 2 diabetes in high-risk individuals. Int. J. Behav. Nutr. Phys. Act. 2017, 14, 78. [CrossRef] [PubMed]

20. Gower, B.A.; Goss, A.M. A lower-carbohydrate, higher-fat diet reduces abdominal and intermuscular fat and increases insulin sensitivity in adults at risk of type 2 diabetes. J. Nutr. 2015, 145, 177s-183s. [CrossRef] [PubMed]

21. Qian, F.; Korat, A.A.; Malik, V.; Hu, F.B. Metabolic Effects of Monounsaturated Fatty Acid-Enriched Diets Compared With Carbohydrate or Polyunsaturated Fatty Acid-Enriched Diets in Patients with Type 2 Diabetes: A Systematic Review and Meta-analysis of Randomized Controlled Trials. Diabetes Care 2016, 39, 1448-1457. [CrossRef] [PubMed]

22. Snorgaard, O.; Poulsen, G.M.; Andersen, H.K.; Astrup, A. Systematic review and meta-analysis of dietary carbohydrate restriction in patients with type 2 diabetes. BMJ Open Diabetes Res. Care 2017, 5, e000354. [CrossRef] [PubMed]

23. Wheeler, M.L.; Dunbar, S.A.; Jaacks, L.M.; Karmally, W.; Mayer-Davis, E.J.; Wylie-Rosett, J.; Yancy, W.S., Jr. Macronutrients, food groups, and eating patterns in the management of diabetes: A systematic review of the literature, 2010. Diabetes Care 2012, 35, 434-445. [CrossRef] [PubMed]

24. Rubino, F.; Forgione, A.; Cummings, D.E.; Vix, M.; Gnuli, D.; Mingrone, G.; Castagneto, M.; Marescaux, J. The mechanism of diabetes control after gastrointestinal bypass surgery reveals a role of the proximal small intestine in the pathophysiology of type 2 diabetes. Ann. Surg. 2006, 244, 741-749. [CrossRef] [PubMed]

25. Urbanova, M.; Mraz, M.; Durovcova, V.; Trachta, P.; Klouckova, J.; Kavalkova, P.; Haluzikova, D.; Lacinova, Z.; Hansikova, H.; Wenchich, L.; et al. The effect of very-low-calorie diet on mitochondrial dysfunction in subcutaneous adipose tissue and peripheral monocytes of obese subjects with type 2 diabetes mellitus. Physiol. Res. 2017, 66, 811-822. [PubMed]

26. Esposito, K.; Maiorino, M.I.; Ciotola, M.; Di Palo, C.; Scognamiglio, P.; Gicchino, M.; Petrizzo, M.; Saccomanno, F.; Beneduce, F.; Ceriello, A.; et al. Effects of a Mediterranean-style diet on the need for antihyperglycemic drug therapy in patients with newly diagnosed type 2 diabetes: A randomized trial. Ann. Intern. Med. 2009, 151, 306-314. [CrossRef] [PubMed]

27. Kahleova, H.; Belinova, L.; Malinska, H.; Oliyarnyk, O.; Trnovska, J.; Skop, V.; Kazdova, L.; Dezortova, M.; Hajek, M.; Tura, A.; et al. Eating two larger meals a day (breakfast and lunch) is more effective than six smaller meals in a reduced-energy regimen for patients with type 2 diabetes: A randomised crossover study. Diabetologia 2014, 57, 1552-1560. [CrossRef] [PubMed]

28. Salas-Salvado, J.; Bullo, M.; Babio, N.; Martinez-Gonzalez, M.A.; Ibarrola-Jurado, N.; Basora, J.; Estruch, R.; Covas, M.I.; Corella, D.; Aros, F.; et al. Reduction in the incidence of type 2 diabetes with the Mediterranean diet: Results of the PREDIMED-Reus nutrition intervention randomized trial. Diabetes Care 2011, 34, 14-19. [CrossRef] [PubMed]

29. Estruch, R.; Ros, E.; Salas-Salvado, J.; Covas, M.I.; Corella, D.; Aros, F.; Gomez-Gracia, E.; Ruiz-Gutierrez, V.; Fiol, M.; Lapetra, J.; et al. Primary prevention of cardiovascular disease with a Mediterranean diet. N. Engl. J. Med. 2013, 368, 1279-1290. [CrossRef] [PubMed]

30. Estruch, R.; Martinez-Gonzalez, M.A.; Corella, D.; Salas-Salvado, J.; Fito, M.; Chiva-Blanch, G.; Fiol, M.; Gomez-Gracia, E.; Aros, F.; Lapetra, J.; et al. Effect of a high-fat Mediterranean diet on bodyweight and waist circumference: A prespecified secondary outcomes analysis of the PREDIMED randomised controlled trial. Lancet Diabetes Endocrinol. 2016, 4, 666-676. [CrossRef]

31. Leslie, W.S.; Taylor, R.; Harris, L.; Lean, M.E. Weight losses with low-energy formula diets in obese patients with and without type 2 diabetes: Systematic review and meta-analysis. Int. J. Obes. (Lond.) 2017, 41, 96-101. [CrossRef] [PubMed]

32. Rehackova, L.; Araujo-Soares, V.; Adamson, A.J.; Steven, S.; Taylor, R.; Sniehotta, F.F. Acceptability of a very-low-energy diet in Type 2 diabetes: Patient experiences and behaviour regulation. Diabet. Med. 2017. [CrossRef] [PubMed]

33. Stefan, N.; Haring, H.U.; Schulze, M.B. Metabolically healthy obesity: The low-hanging fruit in obesity treatment? Lancet Diabetes Endocrinol. 2017. [CrossRef]

34. Banasik, J.L.; Walker, M.K.; Randall, J.M.; Netjes, R.B.; Foutz, M.S. Low-calorie diet induced weight loss may alter regulatory hormones and contribute to rebound visceral adiposity in obese persons with a family history of type-2 diabetes. J. Am. Assoc. Nurse Pract. 2013, 25, 440-448. [CrossRef] [PubMed] 
35. Garcia-Perez, L.E.; Alvarez, M.; Dilla, T.; Gil-Guillen, V.; Orozco-Beltran, D. Adherence to therapies in patients with type 2 diabetes. Diabetes Ther. 2013, 4, 175-194. [CrossRef] [PubMed]

36. Shultz, J.A.; Sprague, M.A.; Branen, L.J.; Lambeth, S. A comparison of views of individuals with type 2 diabetes mellitus and diabetes educators about barriers to diet and exercise. J. Health Commun. 2001, 6, 99-115. [CrossRef] [PubMed]

37. Sellahewa, L.; Khan, C.; Lakkunarajah, S.; Idris, I. A Systematic Review of Evidence on the Use of Very Low Calorie Diets in People with Diabetes. Curr. Diabetes Rev. 2017, 13, 35-46. [CrossRef] [PubMed]

38. Cerrelli, F.; Manini, R.; Forlani, G.; Baraldi, L.; Melchionda, N.; Marchesini, G. Eating behavior affects quality of life in type 2 diabetes mellitus. Eat. Weight Disord. 2005, 10, 251-257. [CrossRef] [PubMed]

39. Carbonneau, E.; Royer, M.M.; Richard, C.; Couture, P.; Desroches, S.; Lemieux, S.; Lamarche, B. Effects of the Mediterranean Diet before and after Weight Loss on Eating Behavioral Traits in Men with Metabolic Syndrome. Nutrients 2017, 9, 305. [CrossRef] [PubMed]

40. Miller, C.K.; Kristeller, J.L.; Headings, A.; Nagaraja, H. Comparison of a mindful eating intervention to a diabetes self-management intervention among adults with type 2 diabetes: A randomized controlled trial. Health Educ. Behav. 2014, 41, 145-154. [CrossRef] [PubMed]

41. Shirai, K.; Saiki, A.; Oikawa, S.; Teramoto, T.; Yamada, N.; Ishibashi, S.; Tada, N.; Miyazaki, S.; Inoue, I.; Murano, S.; et al. The effects of partial use of formula diet on weight reduction and metabolic variables in obese type 2 diabetic patients-multicenter trial. Obes. Res. Clin. Pract. 2013, 7, e43-e54. [CrossRef] [PubMed]

(C) 2018 by the authors. Licensee MDPI, Basel, Switzerland. This article is an open access article distributed under the terms and conditions of the Creative Commons Attribution (CC BY) license (http://creativecommons.org/licenses/by/4.0/). 